\title{
Weakly Self-Avoiding Words and a Construction of Friedman
}

\author{
Jeffrey Shallit* and Ming-wei Wang \\ Department of Computer Science \\ University of Waterloo \\ Waterloo, Ontario, Canada N2L 3G1 \\ shallit@graceland.uwaterloo.ca \\ m2wang@math. uwaterloo.ca
}

Submitted: September 28, 2000; Accepted: February 7, 2001.

MR Subject Classifications: 68R15 Primary

\begin{abstract}
H. Friedman obtained remarkable results about the longest finite sequence $x$ over a finite alphabet such that for all $i \neq j$ the word $x[i . .2 i]$ is not a subsequence of $x[j . .2 j]$. In this note we consider what happens when "subsequence" is replaced by "subword"; we call such a sequence a "weakly self-avoiding word". We prove that over an alphabet of size 1 or 2, there is an upper bound on the length of weakly self-avoiding words, while if the alphabet is of size 3 or more, there exists an infinite weakly self-avoiding word.
\end{abstract}

\section{Introduction}

We say a word $y$ is a subsequence of a word $z$ if $y$ can be obtained by striking out 0 or more symbols from $z$. For example, "iron" is a subsequence of "introduction". We say a word $y$ is a subword of a word $z$ if there exist words $w, x$ such that $z=w y x$. For example, "duct" is a subword of "introduction". ${ }^{1}$

We use the notation $x[k]$ to denote the $k^{\prime}$ th letter chosen from the string $x$. We write $x[a . . b]$ to denote the subword of $x$ of length $b-a+1$ starting at position $a$ and ending at position $b$.

Recently H. Friedman has found a remarkable construction that generates extremely large numbers $[1,2]$. Namely, consider words over a finite alphabet $\Sigma$ of cardinality $k$. If

\footnotetext{
${ }^{*}$ Research supported in part by a grant from NSERC.

${ }^{1}$ Europeans usually use the term "factor" for what we have called "subword", and they sometimes use the term "subword" for what we have called "subsequence".
} 
an infinite word $\mathbf{x}$ has the property that for all $i, j$ with $0<i<j$ the subword $\mathbf{x}[i . .2 i]$ is not a subsequence of $\mathbf{x}[j . .2 j]$, we call it self-avoiding. We apply the same definition for a finite word $x$ of length $n$, imposing the additional restriction that $j \leq n / 2$.

Friedman shows there are no infinite self-avoiding words over a finite alphabet. Furthermore, he shows that for each $k$ there exists a longest finite self-avoiding word $x$ over an alphabet of size $k$. Call $n(k)$ the length of such a word. Then clearly $n(1)=3$ and a simple argument shows that $n(2)=11$. Friedman shows that $n(3)$ is greater than the incomprehensibly large number $A_{7198}(158386)$, where $A$ is the Ackermann function.

Jean-Paul Allouche asked what happens when "subsequence" is replaced by "subword". A priori we do not expect results as strange as Friedman's, since there are no infinite anti-chains for the partial order defined by " $x$ is a subsequence of $y$ ", while there are infinite anti-chains for the partial order defined by " $x$ is a subword of $y$ ".

\section{Main Results}

If an infinite word $\mathbf{x}$ has the property that for all $i, j$ with $0 \leq i<j$ the subword $\mathbf{x}[i . .2 i]$ is not a subword of $\mathbf{x}[j . .2 j]$, we call it weakly self-avoiding. If $x$ is a finite word of length $n$, we apply the same definition with the additional restriction that $j \leq n / 2$.

Theorem 1 Let $\Sigma=\{0,1, \ldots, k-1\}$.

(a) If $k=1$, the longest weakly self-avoiding word is of length 3, namely 000 .

(b) If $k=2$, there are no weakly self-avoiding words of length $>13$. There are 8 longest weakly self-avoiding words, namely 0010111111010, 0010111111011, 0011110101010, 0011110101011 and the four words obtained by changing 0 to 1 and 1 to 0.

(c) If $k \geq 3$, there exists an infinite weakly self-avoiding word.

\section{Proof.}

(a) If a word $x$ over $\Sigma=\{0\}$ is of length $\geq 4$, then it must contain 0000 as a prefix. Then $x[1 . .2]=00$ is a subword of $x[2 . .4]=000$.

(b) To prove this result, we create a tree whose root is labeled with $\epsilon$, the empty word. If a node's label $x$ is weakly self-avoiding, then it has two children labeled $x 0$ and $x 1$. This tree is finite if and only if there is a longest weakly self-avoiding word. In this case, the leaves of the tree represent non-weakly-self-avoiding words that are minimal in the sense that any proper prefix is weakly self-avoiding.

Now we use a classical breadth-first tree traversal technique, as follows: We maintain a queue, $Q$, and initialize it with the empty word $\epsilon$. If the queue is empty, we are done. Otherwise, we pop the first element $q$ from the queue and check to see if it is weakly self-avoiding. If not, the node is a leaf, and we print it out. If $q$ is weakly self-avoiding then we append $q 0$ and $q 1$ to the end of the queue. 
If this algorithm terminates, we have proved that there is a longest weakly self-avoiding word. The proof may be concisely represented by listing the leaves in breadth-first order. We may shorten the tree by assuming, without loss of generality, that the root is labeled 0 .

When we perform this procedure, we obtain a tree with 92 leaves, whose longest label is of length 14. The following list describes this tree:

$\begin{array}{rrrr}0000 & 00111100 & 0011010101 & 001011111011 \\ 0001 & 00111110 & 0011010110 & 001011111100 \\ 0101 & 00111111 & 0011010111 & 00101111110 \\ 001000 & 01000000 & 0011101000 & 001011111111 \\ 001001 & 01000001 & 0011101001 & 001110101000 \\ 001010 & 01000010 & 0011101011 & 001110101001 \\ 001100 & 01000011 & 0011110100 & 001110101010 \\ 010001 & 01100001 & 0011110110 & 001110101011 \\ 010010 & 01100010 & 0011110111 & 001111010100 \\ 010011 & 01100011 & 0110000000 & 001111010110 \\ 011001 & 01110001 & 0110000001 & 001111010111 \\ 011010 & 01110010 & 0110000010 & 011100000000 \\ 011011 & 01110011 & 0110000011 & 011100000001 \\ 011101 & 0010110100 & 0111000001 & 011100000010 \\ 011110 & 0010110101 & 0111000010 & 011100000011 \\ 011111 & 0010110110 & 0111000011 & 00101111110100 \\ 00101100 & 0010110111 & 001011110100 & 00101111110101 \\ 00110100 & 0010111000 & 001011110101 & 00101111110110 \\ 00110110 & 0010111001 & 001011110110 & 00101111110111 \\ 00110111 & 0010111010 & 001011110111 & 00111101010100 \\ 00111000 & 0010111011 & 001011111000 & 00111101010101 \\ 00111001 & 0010111100 & 001011111001 & 00111101010110 \\ 00111011 & 0011010100 & 001011111010 & 00111101010111\end{array}$

Figure 1: Leaves of the tree giving a proof of Theorem 1 (b)

(c) Consider the word

$$
\begin{aligned}
\mathbf{x} & =22010110111011111011111110111111111110 \ldots \\
& =220101^{2} 01^{3} 01^{5} 01^{7} 01^{11} 01^{15} 01^{23} 01^{31} 01^{47} 0 \ldots
\end{aligned}
$$

where there are 0's in positions $3,5,8,12,18,26,38,54,78,110,158, \ldots$ More precisely, define $f_{2 n+1}=5 \cdot 2^{n}-2$ for $n \geq 0$, and $f_{2 n}=7 \cdot 2^{n-1}-2$ for $n \geq 1$. Then $\mathbf{x}$ has 0's only in the positions given by $f_{i}$ for $i \geq 1$.

First we claim that if $i \geq 3$, then any subword of the form $\mathbf{x}[i . .2 i]$ contains exactly two 0 's. This is easily verified for $i=3$. If $5 \cdot 2^{n}-1 \leq i<7 \cdot 2^{n}-1$ and $n \geq 0$, then there are 
0 's at positions $7 \cdot 2^{n}-2$ and $5 \cdot 2^{n+1}-2$. (The next 0 is at position $7 \cdot 2^{n+1}-2$, which is $>2\left(7 \cdot 2^{n}-2\right)$.) On the other hand, if $7 \cdot 2^{n-1}-1 \leq i<5 \cdot 2^{n}-1$ for $n \geq 1$, then there are 0 's at positions $5 \cdot 2^{n}-2$ and $7 \cdot 2^{n}-2$. (The next 0 is at position $5 \cdot 2^{n+1}-2$, which is $>2 \cdot\left(5 \cdot 2^{n}-2\right)$.)

Now we prove that $\mathbf{x}$ is weakly self-avoiding. Clearly $\mathbf{x}[1 . .2]=22$ is not a subword of any subword of the form $\mathbf{x}[j . .2 j]$ for any $j \geq 2$. Similarly, $\mathbf{x}[2 . .4]=201$ is not a subword of any subword of the form $\mathbf{x}[j .2 j]$ for any $j \geq 3$. Now consider subwords of the form $t:=\mathbf{x}[i . .2 i]$ and $t^{\prime}:=\mathbf{x}[j . .2 j]$ for $i, j \geq 3$ and $i<j$. From above we know $t=1^{u} 01^{v} 01^{w}$, and $t^{\prime}=1^{u^{\prime}} 01^{v^{\prime}} 01^{w^{\prime}}$. For $t$ to be a subword of $t^{\prime}$ we must have $u \leq u^{\prime}, v=v^{\prime}$, and $w \leq w^{\prime}$. But since the blocks of 1 's in $\mathbf{x}$ are distinct in size, this means that the middle block of $1^{\prime}$ 's in $t$ and $t^{\prime}$ must occur in the same positions of $\mathbf{x}$. Then $u \leq u^{\prime}$ implies $i \geq j$, a contradiction.

\section{Another construction}

Friedman has also considered variations on his construction, such as the following: let $M_{2}(n)$ denote the length of the longest finite word $\mathbf{x}$ over $\{0,1\}$ such that $\mathbf{x}[i . .2 i]$ is not a subsequence of $\mathbf{x}[j . .2 j]$ for $n \leq i<j$. We can again consider this where "subsequence" is replaced by "subword".

Theorem 2 There exists an infinite word $\mathbf{x}$ over $\{0,1\}$ such that $\mathbf{x}[i . .2 i]$ is not a subword of $\mathbf{x}[j . .2 j]$ for all $i, j$ with $2 \leq i<j$.

Proof. Let

$$
\begin{aligned}
\mathbf{x} & =001001^{3} 01^{2} 01^{7} 01^{5} 01^{15} 01^{11} 01^{31} 01^{23} \ldots \\
& =001001^{g_{1}} 01^{g_{2}} 01^{g_{3}} 0 \cdots
\end{aligned}
$$

where $g_{1}=3, g_{2}=2$, and $g_{n}=2 g_{n-2}+1$ for $n \geq 3$. Then a proof similar to that above shows that every subword of the form $\mathbf{x}[i . .2 i]$ contains exactly two 0 's, and hence, since the $g_{i}$ are all distinct, we have $\mathbf{x}[i . .2 i]$ is not a subword of $\mathbf{x}[j . .2 j]$ for $j>i>1$.

\section{References}

[1] H. Friedman. Long finite sequences. To appear, J. Combinat. Theory A. Also available at <http://www.math.ohio-state.edu/foundations/manuscripts.html>.

[2] H. Friedman. Enormous integers in real life. Manuscript, dated June 1 2000, available at <http://www.math.ohio-state.edu/foundations/manuscripts.html>. 